\title{
DEVELOPMENT OF A HIGH SPECTRAL RESOLUTION LIDAR USING A MULTI-MODE LASER AND A TUNABLE INTERFEROMETER
}

\author{
Pablo Ristori ${ }^{1 *}$, Lidia Otero ${ }^{1-3}$, Yoshitaka Jin $^{2}$, Nobuo Sugimoto ${ }^{2}$, Tomoaki Nishizawa ${ }^{2}$, Eduardo \\ Quel $^{1-3}$ \\ ${ }^{1}$ División Lidar, CEILAP, UNIDEF (MINDEF - CONICET), UMI-IFAECI-CNRS 3351, Juan Bautista de \\ La Salle 4397 -B1603ALO Villa Martelli,Buenos Aires, Argentina, *Email: pablo.ristori@gmail.com \\ ${ }^{2}$ National Institute for Environmental Studies, 16-2 Onogawa, Tsukuba 305-8506 Japan \\ ${ }^{3} I U E$ - Escuela Superior Técnica, Facultad de Ingeniería, Av. Cabildo 15 - C1426AAA CABA, Argentina
}

\begin{abstract}
A High Spectral Resolution Lidar (HSRL) using an unseeded laser is designed to separate Mie from total atmospheric backscatter by means of a tunable interferometer. The separation is achieved by tuning the interferometer's free spectral range (FSR) to longitudinal mode separation of the laser. When this condition is achieved, the interferometer transmits aerosol and molecular backscatter with different efficiencies due to their spectral properties. We estimate those transmissions for the perfect tuning case and introducing accuracy or precision errors on the interferometer alignment.
\end{abstract}

\section{INTRODUCTION}

Conventional aerosol lidar systems collect the atmospheric backscattered radiation from aerosols and molecules at the same wavelength of the laser emission. Many inversion methods [1-5] intend to determine aerosol backscatter and extinction modelling the molecular profile by using the atmospheric temperature and pressure height distribution. This method needs to estimate the aerosol backscatter-to-extinction ratio.

Rotational-vibrational nitrogen and oxygen Raman shifted backscatter returns are not affected by aerosol backscatter and can be combined to the elastic return to determine aerosol backscatter and extinction. However, the Raman cross section is small and the Raman shift may be important. This method needs the extinction spectral dependence (Ångström coefficient) which can be imposed or calculated from other profiles [6]. Pure rotational Raman returns may provide a stronger signal which is spectrally closer to the elastic emission but the signal is very weak.
As the spectral broadening of the laser emission due to the molecular backscatter is much wider than the one from aerosol backscatter, lidars can use this property to separate molecules from aerosol. The laser emission must be such that the broadened returns from molecules and aerosols are spectrally different. Traditional HSRL uses a single mode laser which linewidth is narrower that the molecular backscatter broadening. The collected light passes through a Fabry Perot interferometer (acting as a narrow band-pass filter) or an atomic filter (acting as a notch filter) [7-8] before detection. On this respect, a $60 \mathrm{~cm}$ cavity Nd:YAG seeded laser can provide a linewidth 200 times smaller than an unseeded laser with multiple oscillating modes creating a linewidth of $1 \mathrm{~cm}^{-1}$.

A different HSRL technique using an unseeded multimode laser is proposed on this paper.

\section{METHODOLOGY}

This method intends to separate the aerosol and molecular backscatter returns from the atmospheric backscattered generated by the fine spectral structure of the emission modes of an unseeded laser. The laser cavity generates longitudinal modes equally spaced in the spectrum modulated by the laser gain curve. For example, a $60 \mathrm{~cm}$ effective length cavity will have 120 modes in $1 \mathrm{~cm}^{-1}$. The aerosol backscattered return will have similar spectral signature than the unseeded laser but the molecular backscatter return will be pressure and temperature broadened.

Let' s consider a filter placed at the lidar detection unit which spectral transmission function is periodic and which local maximums (or minimums) coincide with the longitudinal modes 
of the unseeded laser source. This filter will affect considerably the aerosol return, but not the atmospheric-broadened molecular return which will be constant and will have an efficiency equal to the spectrally-integrated filter transmission. The interferometer (Fabry Perot, Michelson, Mach Zehnder) path difference must be equal or multiple (or sub-multiple) of the laser effective cavity length.

The signal obtained for the fundamental or laser harmonics by this channel is represented by eq. 1 :

$$
S_{\text {new }}(r)=a S_{\text {mol }}(r)+b S_{\text {aer }}(r)
$$

where $S_{m o l}$ and $S_{a e r}$ are the range $r$ dependent molecular and aerosol attenuated backscatter returns respectively; the variables $a$ and $b$ are the molecular and aerosol detection efficiencies for this channel. This is a major change from a standard channel, that collects the molecular and aerosol signals with the same efficiency, and makes possible the calculation of separated molecular and aerosol attenuated backscatter independently. If a second detector collecting the complementary light, the system will improve its performance. This can be done using Michelson or Mach Zehnder interferometers. The first consequence of this improvement is that the channel sum provides the total backscattered signal making useless the presence of an additional elastic channel. Furthermore, analyzing equations (2) and (3)

$$
\begin{aligned}
& S_{n e w_{1}}(r)=\frac{1}{2} S_{m o l}(r)+\left(\frac{1+c}{2}\right) S_{a e r}(r) \\
& S_{n e w_{2}}(r)=\frac{1}{2} S_{m o l}(r)+\left(\frac{1-c}{2}\right) S_{a e r}(r)
\end{aligned}
$$

it can be seen that the subtraction of (2) from (3) (after compensating for different detection efficiencies) gives the aerosol backscatter return as seen on equation (4)

$$
c S_{\text {aer }}(r)=S_{n e w_{1}}(r)-S_{n e w_{2}}(r)
$$

This is the basic concept of the multi-mode High Spectral Resolution Lidar (MM-HSRL).

Reference [10] shows a direct detection wind lidar that uses a multimode laser and a quadri-channel Mach-Zehnder interferometer to measure aerosol wind speed extracted from its Doppler-shifted return.

\section{ANALYSIS}

To start the analysis prior to the construction we must take into account several conditions. This work study the interferometer misalignment effects while [9] studies comes other issues like angular alignment and some laser emission properties.

This system is very sensible to the interferometer path difference. Solving the aerosol equation like in (4) when this path is not the optimum can lead to an ill posed problem adding noise or making impossible to reach to a solution. We use the matrix condition number (maximum to minimum singular value ratio) as an indicator of the stability of this inversion.

We will study the alignment problem considering first the effect of the precision analyzing the signal behavior close to the absolute maximum, and then the effect of the accuracy analyzing the local maximum intensities as a function of its distance to the absolute maximum.

For this analysis a simulation was done using the following parameters:

- The laser considered was a Nd:YAG with maximum gain set at $1064.15 \mathrm{~nm}$

- Laser gain curve was simulated to have Gaussian shape of $1 \mathrm{~cm}^{-1} \mathrm{FWHM}$

- The cavity effective length was considered to be $48.5 \mathrm{~cm}$ (FSR $=0.0104$ $\mathrm{cm}^{-1}$ ) with a front mirror reflectivity equal to $16 \%$

- The atmospheric molecular broadening was set $3 \mathrm{GHz}$

- No broadening nor wavelength shift was considered for the aerosol backscatter

- The interferometers simulated were Fabry Perot (reflectivity 40\%) and Michelson (or Mach Zehnder)

\section{RESULTS}

\subsection{System Precision}

If the interferometer is close to its best alignment position (accurate alignment) then it has reached a region at which minor movements create an important change in the intensity of the 
transmitted aerosol backscatter radiation. Then a precise alignment is required. For the analysis simulation the ratio from the maximum to the minimum is about 2.33 and 1.91 for Michelson and Fabry Perot respectively. Figure 1 shows the percentage of the backscatter intensity transmitted by the interferometers from molecular and aerosol backscatter returns. Figure 2 show the condition number (maximal to minimal singular value ratio) where larger is worst.
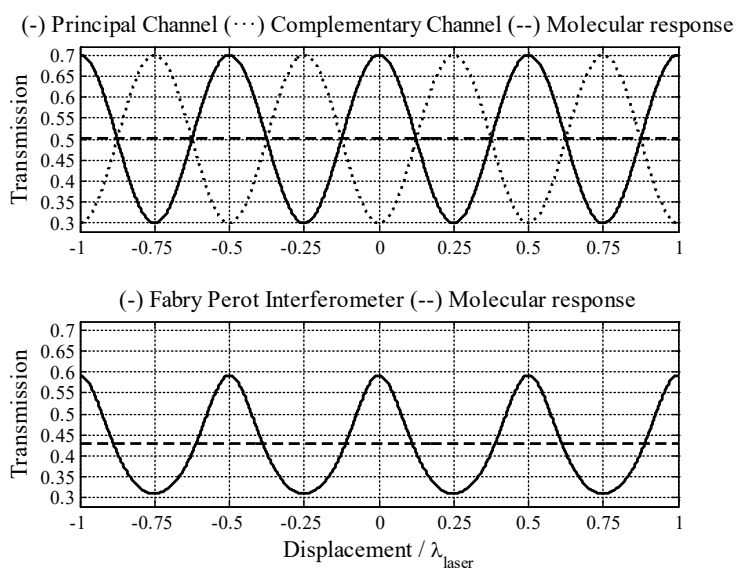

Figure 1: Transmission efficiency: (upper panel) for the Principal and Complementary aerosol channels of Michelson / Mach-Zehnder Interferometer compared to the molecular transmission efficiency which in both cases is 0.5; (Lower panel) for Fabry Perot aerosol and molecular channels
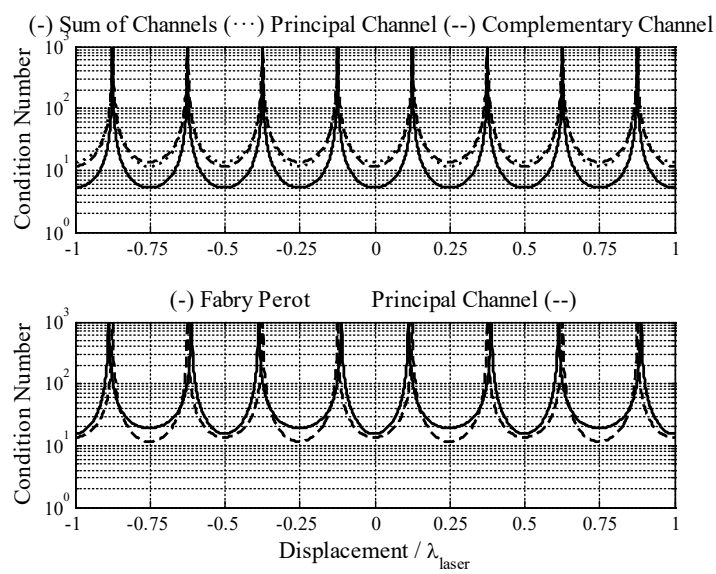

Figure 2: Condition number for (upper panel) Michelson/Mach-Zehnder Interferometer: Principal and Complementary Channel in dashed and dotted lines; combination in continuous line; (lower panel) Condition number comparison between Principal Channel above and Fabry Perot channel

\subsection{System Accuracy}

Even if the interferometer may track a local maximum, it may be still far from the absolute maximum (lack of accuracy). As for the system precision study, Figure 3 shows the percentage of the backscatter intensity from molecules and particles in a system which is precisely aligned to its local maximum as a function of its displacement to the absolute maximum.
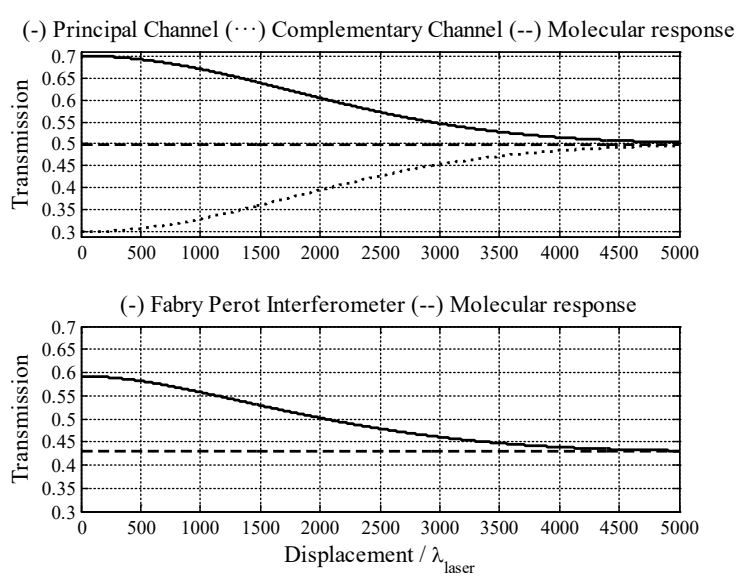

Figure 3: Same as Figure 1 but following the maximum transmission up to $5000 \lambda_{\text {laser }}$

From these curves it is clear that the accuracy of the simulated system should be better that $\pm 0.1 \%$ of the cavity length $( \pm 1 \mathrm{~mm})$ to have a $10 \%$ reduction of the aerosol signal.

Figure 4 shows the condition number. Using both channels of the Michelson interferometer leads always to better accuracy in the calculation.
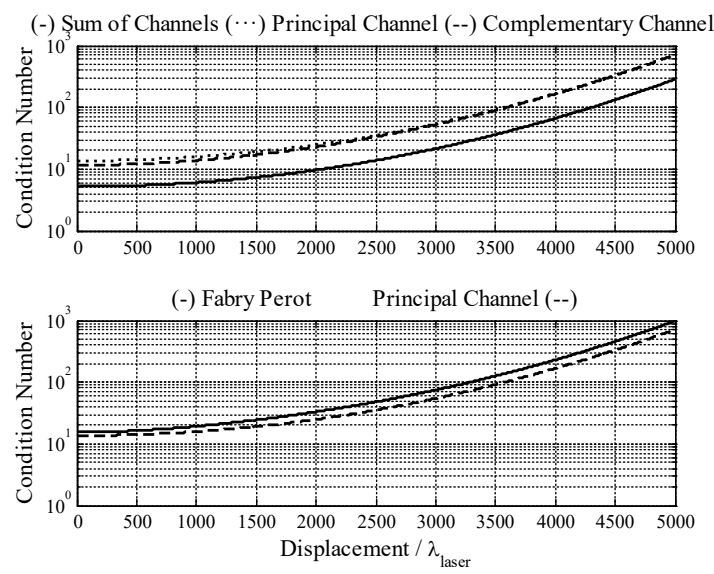

Figure 4: Same as Figure 2 but following the maximum transmission up to $5000 \lambda_{\text {laser }}$ 


\section{CONCLUSIONS}

This work shows to the authors' knowledge the first study of a MM-HSRL for aerosol observations. It important to notice that the emission complexity is similar to traditional aerosol lidar systems and that the detection is not so complex as the standard Fabry Perot based HSRL. To use this technique, the alignment to reach a local maximum and to stay closer as possible to the absolute maximum are critical issues to address.

\section{ACKNOWLEDGEMENT}

The researchers want to acknowledge the Japan Science and Technology Agency (JST) / Japan International Cooperation Agency (JICA), Science and Technology Research Partnership for Sustainable Development (SATREPS).

\section{REFERENCES}

[1] Klett, J. D., 1981: Stable analytical inversion solution for processing lidar returns. App. Opt., 20(2), 211-220.

[2] Fernald, F. G., 1984: Analysis of atmospheric lidar observations- Some comments. App. Opt., 23(5), 652-653.

[3] Wagner, J., A. Ansmann, U. Wandinger, P. Seifert, A. Schwarz, Matthias Tesche, A. Chaikovsky, and O. Dubovik, 2013: Evaluation of the Lidar/Radiometer Inversion Code (LIRIC) to determine microphysical properties of volcanic and desert dust. Atm. Meas. Tech. 6 (7), 17071724.

[4] Ansmann, A., P. Seifert, M. Tesche, and U. Wandinger, 2012: Profiling of fine and coarse particle mass: case studies of Saharan dust and Eyjafjallajökull/Grimsvötn volcanic plumes, Atmos. Chem. Phys., 12, 9399-9415, doi:10.5194/acp-12-9399-2012.

[5] A. Shimizu, N. Sugimoto, I. Matsui, K. Arao, I. Uno, T. Murayama, N. Kagawa, K. Aoki, A. Uchiyama, and A. Yamazaki, 2004: Continuous observations of Asian dust and other aerosols by polarization lidar in China and Japan during ACEAsia, J. Geophys. Res., 109, D19S17, doi:10.1029/2002JD003253.

[6] Otero L., P. Ristori, E. Quel, 2008: Multiwavelength Aerosol and Water Vapor Raman Lidar System at CEILAP, Buenos Aires,
Argentina. Case Study: November 10, 2006. Opt. Pura Apl., 41 (2) 171 - 175,

[7] Shimizu H., S. A. Lee, and C. Y. She, 1983: High spectral resolution lidar system with atomic blocking filters for measuring atmospheric parameters, Appl. Opt., 22, 1373-1381.

[8] Nishizawa T., N. Sugimoto, and I. Matsui, 2010: Development of multi-wavelength highspectral resolution lidar system $(2 \alpha+3 \beta+2 \delta)$, Proc. of 25th International Laser Radar Conference, 903-906.

[9] Jin Y., N. Sugimoto, T. Nishizawa, P. Ristori, and L. Otero, 2015: A concept of multi-mode high spectral resolution lidar using Mach-Zehnder interferometer, Proc. of 27th International Laser Radar Conference.

[10] Bruneau, D., F. Blouzon, J. Spatazza, F. Montmessin, J. Pelon, B. Faure, 2013: Directdetection wind lidar operating with a multimode laser, Appl. Opt., 52, 4941. 